\title{
Physik in unserer Zeit
}




\title{
Sind Dauerströme noch normal?
}

\author{
Ulrich Eckern
}

\section{Elektrische Dauerströme, hervorstechendes Merkmal supraleitender Materialien, sind auch in kleinen - mesoskopischen - Normalmetall- und Halbleiterringen beobachtbar. Neueste Experimente, die bei Temperaturen in Nähe des absoluten Nullpunktes durchgeführt wurden, lassen sich nur mit Hilfe der Wellennatur der Elektronen erklären und eröffnen überraschende Einsichten in grundlegende Fragen der Quantenmechanik.}

\begin{abstract}
$\mathrm{I}_{\mathrm{m}}^{\mathrm{m}}$ Zuge voranschreitender Miniaturisierung elektronischer Bauelemente erschließt sich dem Festkörperphysiker ein Grenzbereich, mesoskopische Physik genannt, in dem neuartige Phänomene von sowohl grundsätzlicher als auch technologischer Bedeutung auftreten. Grob gesprochen ist das Verhalten der Elektronen im makroskopischen Bereich, etwa in kommerziell erhältlichen elektronischen Bauelementen, weitgehend mit Hilfe klassischer Transporttheorien erklärbar. Andererseits ist seit den zwanziger Jahren dieses Jahrhunderts wohlbekannt, daß Elektronen in mikroskopischen Systemen wie Atomen und Molekülen durch eine komplexwertige quantenmechanische Wellenfunktion beschrieben werden müssen. Hier führt die Lösung der Schrödinger-Gleichung mit bestimmten Randbedingungen unter anderem zu dem Ergebnis, daß nur diskrete Werte der Energie möglich sind.
\end{abstract}

Mesoskopische Systeme, die in einem Zwischenbereich liegen, haben typische räumliche Abmessungen im Bereich von zehn Nanometern bis zu einigen Mikrometern. In Experimenten bei tiefen Temperaturen, etwa unterhalb $1 \mathrm{~K}$, zeigen solche Systeme bemerkenswerte Abweichungen vom klassischen Verhalten. Ein Beispiel ist die Möglichkeit, mit Hilfe sehr kleiner Tunnelkontakte einzelne Elektronen zu manipulieren [1]. Die Wellennatur der Teilchen beinhaltet darüber hinaus die Möglichkeit von Interferenzen, wie sie aus der Physik klassischer Wellen wohlbekannt sind.

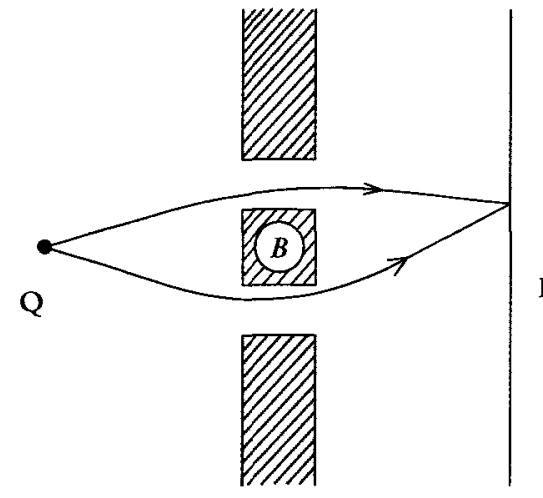

D

Abb. 1. Ideale Doppelspaltgeometrie für den Aharonov-Bohm Effekt. Das Magnetfeld steht senkrecht zur Ebene und ist auf den Bereich B beschränkt. Die Elektronen werden durch das Feld nicht beschleunigt.

Thema des vorliegenden Beitrages sind die schon seit langem diskutierten, aber erst vor etwa vier Jahren experimentell nachgewiesenen Dauerströme in kleinen metallischen Ringen, etwa aus Gold oder Kupfer, die letztlich mit den bereits erwähnten quantenmechanischen Interferenzen zusammenhängen und die durch einen magnetischen Fluß vom Experimentator gesteuert werden können (Aharonov-Bohm Effekt).

Diese Dauerströme lassen sich nicht im Rahmen der klassischen Physik erklären - in diesem Sinne sind sie unnormal. Aber auch bei quantenmechanischer Betrachtung herrschte lange Zeit die Meinung vor, daßs die bei der
Herstellung von metallischen Ringen (siehe Informationskasten in [1]) notwendigerweise auftretenden, zufällig angeordneten Defekte den Effekt unbeobachtbar machen müßten. Wie sich aber herausgestellt hat, war das nicht richtig, „normale“ Dauerströme wurden tatsächlich experimentell nachgewiesen [2-4] - und die beobachteten Effekte sind dabei deutlich größer als von neuen, sogar verbesserten Theorien vorhergesagt wurde [5-8]!

\section{Dauerströme in Supraleitern}

Im Gegensatz zu den elektrischen Dauerströmen in Normalmetallen sind sie in supraleitenden Materialien seit mehreren Jahrzehnten wohlbekannt. Supraleiter unterhalb ihrer Übergangstemperatur zeigen ideale Leitfähigkeit und perfekten Diamagnetismus. Das Verschwinden des elektrischen Widerstands läßt sich am genauesten mit Hilfe eines makroskopisch großen Rings demonstrieren. Der Ring wird in einem Magnetfeld von dem Normalin den supraleitenden Zustand abgekühlt. Der nach Abschalten des Feldes durch Induktion angeworfene Dauerstrom kann experimentell beobachtet werden, wobei sich, je nach Dicke des Rings, für den zeitlichen Zerfall des Stromes ohne weiteres Zeitkonstanten von vielen Jahrtausenden (!) ergeben können. In einem vergleichbaren Kupferring, der nicht supraleitend ist, klingt ein so erzeugter "Dauerstrom" aber bereits nach wenigen Mikrosekunden ab - diese Art von Dauerströmen sind nicht Thema des vorliegenden Beitrages! 
Als Folge des perfekten Diamagnetismus wird aber nicht nur das Eindringen eines Magnetfeldes in ein supraleitendes Metall verhindert, sondern das Feld wird auch aus dem Metall beim Eintritt in den supraleitenden Zustand verdrängt. Dies ist der MeissnerOchsenfeld-Effekt. In einer dünnen Oberflächenschicht fließen dabei elektrische Abschirmströme, so lange das Magnetfeld angelegt ist. Es handelt sich um einen stromtragenden Gleichgezichtszustand. In diesem Sinne wollen wir den Begriff Dauerstrom im folgenden gebrauchen.

Mikroskopisch betrachtet bilden die Elektronen in einem Supraleiter einen kohärenten Zustand, in dem alle Elektronen zu Paaren gebunden sind. Die Paare besetzen alle den gleichen quantenmechanischen Zustand. Als Folge ist der Suprazustand außerordentlich robust. Dies trifft aber auf Normalmetallringe nicht $\mathrm{zu}$, hier ist jedes Elektron in einem anderen Zustand, und wir können Dauerströme, wenn überhaupt, nur unter viel einschneidenderen Bedingungen erwarten.

\section{Quanteninterferenzen in metalli- schen Ringen}

Eine idealisierte Anordnung für die Beobachtung von Interferenzen ist in Abbildung 1 gezeigt. Eine Elektronenwelle, die von einer Quelle Q erzeugt wird, breitet sich durch eine Wand mit zwei Spaltöffnungen aus und wird auf einem Schirm D nachgewiesen. Im Bereich $B$ ist ein magnetischer Fluß $\Phi$ eingeschlossen, der so sorgfältig abgeschirmt wird, daß die Elektronen durch das zugehörige magnetische Feld (senkrecht zur gezeichneten Ebene gerichtet) keine Kräfte erfahren. Trotzdem zeigt sich im Experiment, daß das Interferenzmuster auf dem Schirm durch Änderung des magnetischen Flusses verändert werden kann!

Die Erklärung für dieses Phänomen, das nach den Autoren der 1959 erschienenen Arbeit Aharonov-Bohm-Effekt genannt wird, kann nur die Quantenmechanik liefern. Die Phase der Elektronenwellenfunktion wird direkt vom Vektorpotential beeinflußt, und zwar so, daß die Phasendifferenz $\Delta \varphi$ der beiden gezeigten Wege (siehe "Wellenfunktion und Vektorpotential ${ }^{\text {") }}$ durch den eingeschlossenen magnetischen Fluß gegeben ist, nämlich durch

$\Delta \varphi=2 \pi \frac{e}{b} \Phi$

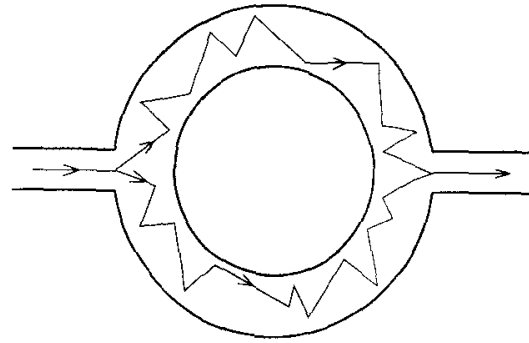

Abb. 2. Aharonov-Bohm Effekt in einem Metallring. Aufgrund elastischer Streuung an Defekten ist die Bewegung der Elektronen diffusiv. Trotzdem sind Interferenzen im Widerstand beobachtbar.

Der Quotient aus Planckschem Wirkungsquantum, $b$, und Elementarladung, $e, \Phi_{0}=$ $b / e$, wird als magnetisches Flußquantum bezeichnet. Der numerische Wert des Flußquants ist $\Phi_{0} \approx 4 \cdot 10^{-11}$ Tesla $\mathrm{cm}^{2}$ (1 Tesla = $10^{4}$ Gauß). Daher ist die obige Phasendifferenz nichts anderes als die Zahl der von den Wegen eingeschlossenen Flußquanten $\Phi / \Phi_{0}$ multipliziert mit $2 \pi$. Da eine Phasenänderung

\section{Wellenfunktion und Vektorpotential}

In der klassischen Mechanik stehen die Bahn eines Teilchens und die Kräfte, die auf das Teilchen wirken, im Zentrum des Interesses. Dieses Konzept wird in der Quantenmechanik Wahrscheinlichkeitsamplituden oder Wellenfunktionen, $\psi(r, t)$, ersetzt. Sie hängen vom Ort und von der Zeit ab. Die Impulse und die Energien, die die Phase der Wellenfunktionen bestimmen, sind die entscheidenden Größen in der quantenmechanischen Theorie. Entsprechend sind es auch das Vektorpotential A und das skalare Potential, und nicht das magnetische und das elektrische Feld, die direkt in die Quantenmechanik eingehen.

Wir beschreiben jetzt kurz das Gesetz, das in der Quantenmechanik das klassische Kraftgesetz $\mathbf{F}=q v \times \mathbf{B}$ ( $q$ ist die Ladung des Teilchens, v seine Geschwindigkeit und B das Magnetfeld) ersetzt. Es ist für das Verständnis von Quanteninterferenzexperimenten von entscheidender Bedeutung. Die Phasenänderung der Wellenfunktion entlang eines bestimmten Weges aufgrund eines magnetischen Feldes ist durch das Integral des Vektorponentials entlang dieses Weges, multipliziert mit $q / \hbar$, gegeben, von $2 \pi$ nicht beobachtbar ist, folgt sofort, daß das Interferenzmuster eine periodische Funktion von $\Phi$ ist, mit Periode $\Phi_{0}$. Dies ist der Aharonov-Bohm-Effekt im Vakuum.

Aber sind denn die Interferenzen auch in metallischen Ringen genügend groß, um beobachtbar zu sein? Ein naheliegendes Experiment ist die Untersuchung des elektrischen Widerstands eines metallischen Rings. Es treten im wesentlichen zwei Schwierigkeiten auf. Zum einen ist da die Temperatur der Elektronen, die dafür sorgt, daß die Zustände der Elektronen miteinander vermischt werden.

Dies geschieht beispielsweise durch Streuung an Gitterschwingungen, die das "Phasengedächtnis" einer Welle zerstören. Bei genügend tiefen Temperaturen, etwa unterhalb $1 \mathrm{~K}$, ist dies jedoch vernachlässigbar, wenn der Ring lediglich einen Umfang von einigen Mikrometern besitzt. Zum anderen enthalten metallische Ringe aber auf jeden Fall - Ausnahmen gibt es nur bei Halbleitern, siehe unten - eine

$$
\varphi_{\text {Weg }}=\frac{q}{\hbar} \int_{\text {Weg }} A \cdot d s
$$

(The Feynman Lectures on Physics, Vol., II Chapter 15-5, Addison-Wesley 1964).

Wenden wir diese Vorschrift auf die in Abbildung 1 gezeigte Geometrie an, und bezeichnen wir den „oberen" Weg mit I und den „unteren ${ }^{*}$ Weg mit II, ergibt sich

$$
\varphi_{I, I I}=\frac{q}{\hbar_{1, I I}} \int_{\mathrm{A}} \mathrm{d} \cdot \mathrm{ds}
$$

Das auf dem Schirm D sichtbare Interferenzmuster hängt von der Phasendifferenz $\Delta \varphi=\varphi_{r} \varphi_{I I}$ ab. Das Negative von $\varphi_{I I}$ ist aber gerade die Phasenänderung entlang des umgekehrt durchlaufenen Weges $I I$, so daß sich das Umlaufintegral

$$
\Delta \varphi=\frac{q}{h} \oint A \cdot d s
$$

ergibt. Dieser Ausdruck läßt sich mit Hilfe des Stokes'schen Satzes in ein Flächenintegral über das Magnetfeld $B=\nabla \times A$ umwandeln, das gleich dem magnetischen Fluß $\Phi$ ist, woraus wir schließlich die Beziehung $\Delta \varphi=(q / n) \Phi$ erhalion. 
große Zahl von Fremdatomen, Fehlstellen und Defekten, bedingt durch den Herstellungsprozeß, die aber im Gegensatz zu den bereits erwähnten Gitterschwingungen weitgehend statisch sind. Streuung an statischen Defekten, obwohl dadurch die Bewegung der Elektronen ähnlich wie bei der Brownschen Bewegung diffusiv wird (Abbildung 2), führt zwar zu einer Phasenverschiebung, nicht aber zur Zerstörung der Phasenkohärenz. Dies konnte experimentell durch Messung des Widerstands als Funktion des Magnetfeldes bestätigt werden. Die Periode im magnetischen Fluß wurde aus der bekannten Geometrie des Rings berechnet, sie beträgt $\Delta \Phi=b / e$. Der Effekt ist jedoch sehr klein. Der Widerstand ist fast vollständig durch das diffuse (klassische) Verhalten der Elektronen bestimmt, und die magnetfeldabhängige Veränderung ist nur eine Korrektur von der Größenordnung weniger Promille. Um den Effekt experimentell beobachtbar zu machen, war es außerdem notwendig, den Ring bis zu einer Temperatur von etwa $10 \mathrm{mK}$, also fast bis zum absoluten Nullpunkt (!), abzukühlen.

Bemerkenswert ist weiterhin, daß der Widerstand und, wie unten beschrieben, auch der Dauerstrom in diesem Temperaturbereich durch mikroskopisch kleine Veränderungen der Defektkonfiguration drastisch verändert werden können. Schon durch Verschieben der Position eines Störatoms um etwa $1 \AA$ können sich Änderungen des Widerstands um ungefähr $b / e^{2} \approx 26 \mathrm{k} \Omega$ ergeben! Zum Verständnis der Physik mesoskopischer Systeme genügt es somit nicht, den Mittelwert physikalischer Größen über mehrere Proben anzugeben genausowenig, wie sich beispielsweise die Ergebnisse beim Würfelspiel durch Angabe des Mittelwerts aller möglichen Würfe $(=3,5)$ charakterisieren lassen!

\section{Experimentelle Beobachtungen}

Nach der bisherigen Diskussion ist klar, daß, wenn überhaupt, Dauerströme nur in winzigen Metallringen und nur bei sehr tiefen Temperaturen beobachtbar sind. Die Experimente, die sich sehr viel leichter beschreiben als tatsächlich durchführen lassen, führen in vieler Hinsicht an die Grenzen des heute technisch Machbaren, sowohl bei der Herstellung der Ringe mit Hilfe modernster lithografischer Techniken, als auch bei der erforderlichen Kühltechnik. Hauptproblem ist jedoch die Tatsache, daß ein außerordentlich kleines magnetisches Signal gemessen werden muß. Vergleichsweise riesige, aber unerwünschte

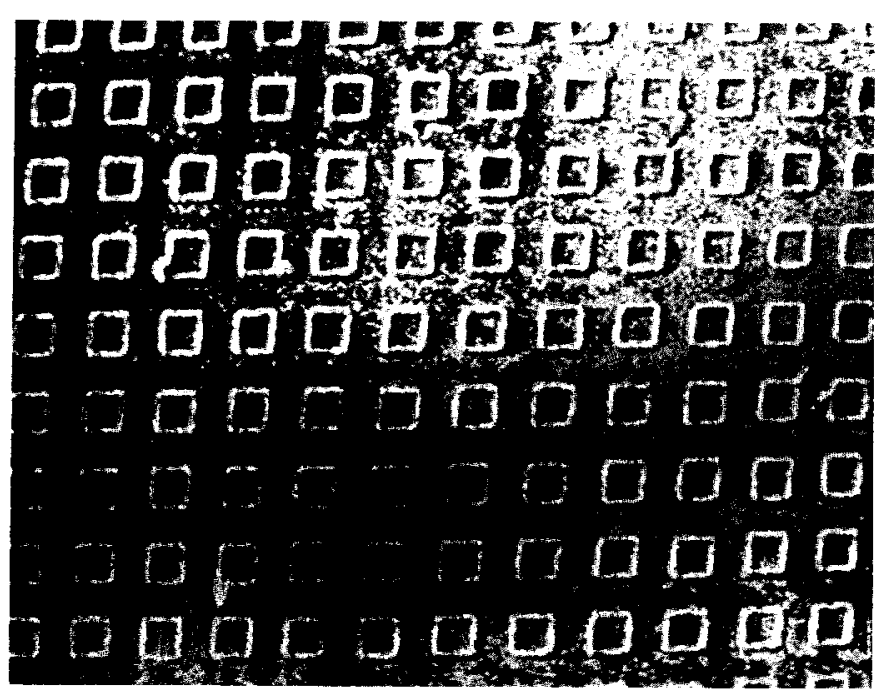

Abb. 3. Elektronenmikroskop-Aufnahme eines Teils der 10 Millionen Cu-,Ringe“ aus [2]. Der Umfang eines Rings ist $\approx \mathbf{2 , 2} \mu \mathrm{m}$, Dicke und Höhe der Drähte ist $\approx 0,04 \mu \mathrm{m}$.

Beiträge der Meßapparatur sowie Beiträge der Eigendrehimpulse der Elektronen müssen dazu herausgefiltert werden.

Das letzte Problem läßt sich aber mit Hilfe eines Tricks lösen, da der erwartete Dauerstrom eine periodische Funktion des angelegten magnetischen Flusses ist. Dazu arbeitet der Experimentator mit einer differentiellen Technik, bei der einem konstanten Magnetfeld ein nicht zu kleines, zeitlich veränderliches Feld überlagert wird. Die höheren Fourierkomponenten des Meßsignals werden dann beobachtet. Zur weiteren Verstärkung des winzigen Signals wurde weiterhin in dem ersten tatsächlich gelungenen Experiment [2] eine riesige Anzahl (ungefähr 10 Millionen) nahezu identischer „Ringe" (siehe Abbildung 3) untersucht, so daß sich das Signal mit Hilfe eines kommerziell erhältlichen SQUID (Superconducting Quantum Interference Device)-Magnetometers nachweisen läßt. Einige Ergebnisse sind in Abbildung 4 gezeigt. Deutlich ist eine Veränderung des Meßsignals erkennbar, die jedoch zu einer Periode von $\Phi_{0} / 2$ (und nicht $\Phi_{0}$ ) gehört. Die Größe des gemessenen Dauerstroms liegt nur bei etwa einem milliardstel Ampere.

Wie können wir die Periodizität $\Phi_{0} / 2$ verstehen, wo doch nach unseren Überlegungen im zweiten Abschnitt die führende Periodizität $\Phi_{0}$ sein sollte? Dazu ist zu beachten, daß das Experiment an vielen Ringen durchgeführt wurde. Es wird also ein Mittelwert gebildet. Wir müssen schließen, daß zwar für einen einzelnen Ring der erste Term in einer Entwicklung nach Harmonischen (siehe „Normale Dauerströme“, Seite 157) ungleich Null ist, aber möglicherweise das Vorzeichen von Ring zu Ring variiert, so daß im Mittel die Periodizität $\Phi_{0}$ nicht beobachtbar ist. Dies wird durch theoretische Untersuchungen erhärtet, die im Mittel über eine Gesamtheit von Ringen nur einen nicht verschwindenden Beitrag mit der Periode $\Phi_{0} / 2$ vorhersagen. Ähnliche Beobachtungen wurden auch im Zusammenhang mit dem Widerstand gemacht. Nach Mittelung über viele Ringe hat der führende Beitrag dort ebenfalls die Periode $\Phi_{0} / 2$.

Damit lag es nahe, in einem weiteren Schritt zur Bestätigung dieser Überlegungen den Versuch zu unternehmen, auch an einzelnen Ringen den Dauerstrom nachzuweisen. Dies ist in der Tat an einzelnen Goldringen [3] und an einem Ring, der auf der Grundlage einer GaAs-GaAlAs Heterostruktur hergestellt wurde [4], gelungen (Abbildungen 5, 6). Die außerordentlichen experimentellen Schwierigkeiten werden unter anderem auch dadurch deutlich, daß die in [2] und [3] beschriebenen Experimente bisher nicht erfolgreich wiederholt werden konnten!

Die Ergebnisse zeigen ganz klar eine Periodizität $\Phi_{0}$ (Abbildung 5), wie erwartet. Überraschend ist jedoch die Größe des Effekts, zumindest für die metallischen Ringe aus [3], in denen eine sehr große Zahl von Streuungen an Defekten stattfindet, wenn eine Elektronenwelle einmal um den Ring herumläuft. Nicht so überraschend war das Ergebnis für die Halbleiter-Heterostruktur. Hier ist die mittlere freie Weglänge, also die Strecke, die ein Elektron auf einer geraden Bahn zwischen zwei Streuprozessen zurücklegt, ungefähr vergleichbar mit dem Umfang des Ringes.

Die Halbierung der Periode bei einer Messung an einer statistischen Gesamtheit von Ringen im Vergleich zu einem einzelnen Ring ist daher eine wohlverstandene Folge der Mittelung. Im Zusammenhang mit der Größe des Effekts sind aber noch fast alle Fragen offen. 


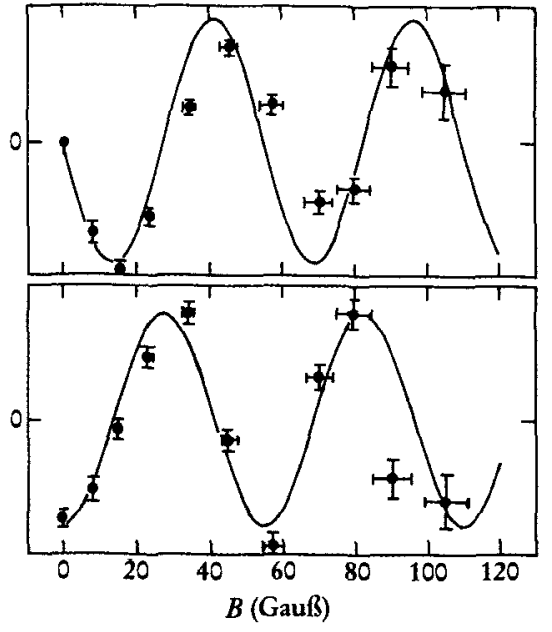

Abb. 4. Die zweite (oberes Bild) und die dritte (unteres Bild) Ableitung der Magnetisierung nach dem Magnetfeld zeigen deutlich eine periodische Abhängigkeit (aus [2]; $T<10 \mathrm{mK}$ ). Die Periode ergibt sich mit Hilfe der Geometrie der Ringe zu $b / 2 e$.

\section{Theoretische Überlegungen}

\section{Ideale Ringe}

Für einen idealen, eindimensionalen Ring ohne Defekte läßt sich das quantenmechanische Energiespektrum als Funktion des magnetischen Flusses und der zugehörige Dauerstrom leicht bestimmen (siehe "Quantenmechanik eines eindimensionalen Ringes", Seite 156). Der Dauerstrom für $\Phi \rightarrow 0$ wird im wesentlichen von dem Elektron mit der höchsten Energie bestimmt - in diesem Sinne haben wir auch hier einen Einzelelektroneneffekt [1] vorliegen. Ohne näher auf die funktionale Form der Flußabhängigkeit einzugehen - der typische Wert des Dauerstroms ist durch das Verhältnis aus mittlerem Energieniveauabstand (in der Nähe des höchsten besetzten Energieniveaus), $\Delta_{0}$, zum Flußquant $\Phi_{0}$ gegeben. Für einen Ring mit $L \approx 10$ $\mu \mathrm{m}$ ergibt sich $\Delta_{\mathrm{C}} \approx 0,1 \mathrm{meV}$, und damit ein Strom von etwa einem hundertstel Mikroampere, überraschenderweise in recht guter Übereinstimmung mit den Experimenten [3, 4] an einzelnen Ringen. Die entsprechende Magnetisierung ist nur etwa tausendmal so groß wie das Bohrsche Magneton, also das
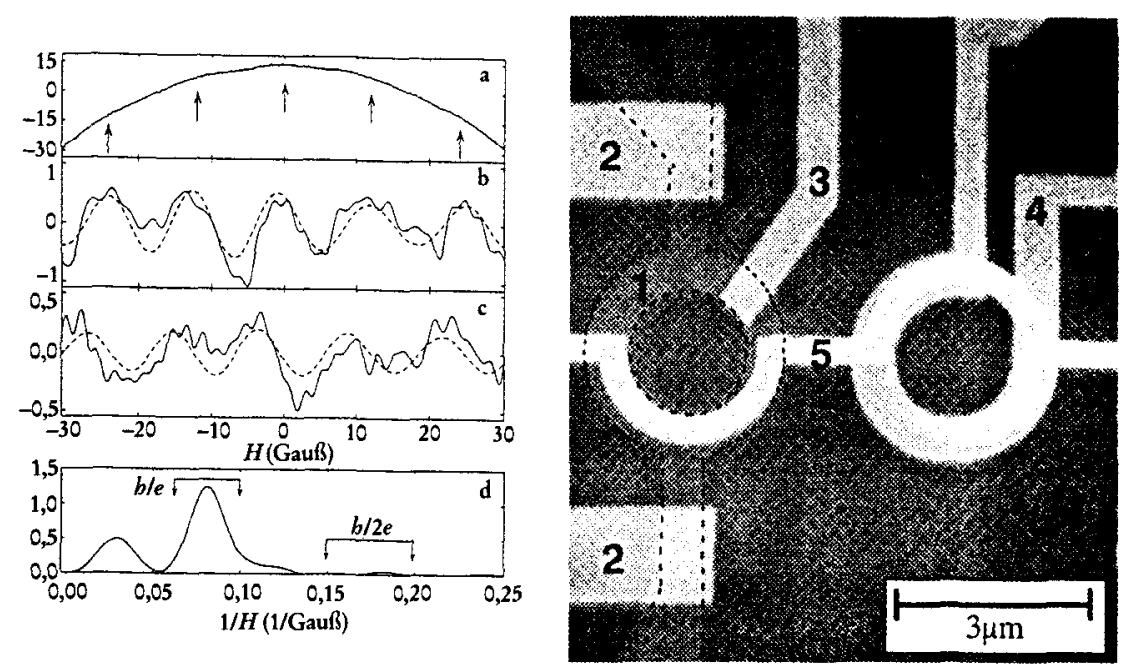

Abb. 5. (a) Erste Ableitung der Magnetisierung nach dem Feld für einen einzelnen Ring, die nach Subtraktion eines quadratischen Hintergrundes (b) deutlich periodisch ist, in Übereinstimmung mit der unabhängig gemessenen zweiten Ableitung (c). (d) Fourier-Leistungsspektrum von (b) nach Glättung, wie dort durch die gestrichelte Linie angedeutet. Die dominante Periode im Fluß ist b/e $(T=7,6 \mathrm{mK}$, Umfang ungefähr $8 \mu \mathrm{m}$; aus [3]).

magnetische Moment eines Elektrons aufgrund seines Eigendrehimpulses. Der Dauerstrom erzeugt im Zentrum des Rings ein Magnetfeld von $10^{-5}$ Gauß. Dies ist fünf Größenordnungen kleiner als das Magnetfeld der Erde, und fast sieben Größenordnunger kleiner als das im Experiment angelegte Meßsfeld. In der Tat ist der Dauerstrom nur ein winziger, quantenmechanischer Effekt, aber einer von grundlegender Bedeutung für unser Verständnis der Quantenmechanik. Anders als bei Dauerströmen in Supraleitern sind Rückwirkungen des angeworfenen Stroms auf das äußere Feld bei Normalmetallringen vollständig vernachlässigbar.

\section{Reale Ringe}

Wie ändert sich nun die theoretische Beschreibung für reale Ringe? Mehrere Schwierigkeiten treten auf. Die kleine, an atomaren Abständen gemessen jedoch große Querschnittsfläche der Drähte führt zunächst dazu, daß etwa zehntausend sogenannte transversale Kanäle, die ungefähr den zu der endlichen Querschnittfläche gehörenden Eigenmoden der elektronischen Zustände entsprechen, zum Strom beitragen. Diese Beiträ-
Abb. 6. Elektronenmikroskop-Aufnahme des Halbleiterrings, der aus einer GaAs/ GaAlAs Heterostruktur hergestellt wurde (1) (die gestrichelte Linie ist nur zur Verdeutlichung eingezeichnet), mit zwei Gates (2) und (3). Rechts befindet sich die Kalibrierungsspule (4). Auf der Anordnung ist die erste Stufe des SQUID (5) mit zwei brückenartigen Josephson-Kontakten (rechts) erkennbar. Die Aufnahme zeigt die Struktur vor der zweiten Stufe der SQUID-Herstellung.

ge können positiv oder negativ sein, so dal? sich durch Addition ein typischer Wert ergibt, der etwa $100 \mathrm{mal}$ größer ist wie beim idealen Ring. Diese Erhöhung wird aber schon durch eine geringe Zahl von Defekten wieder aufgehoben, so daß in der Tat ein Wert $\approx \Delta_{0} / \Phi_{C}$ für dic fast defektfreien Halbleiterringe [4] vernünftig erscheint - in guter Übereinstimmung mit dem Experiment.

Völlig anders ist die Situation in den metallischen Ringen $[2,3]$. Hier ist die mittlere freic Weglänge nur etwa cin Hundertstel des Umfangs, die Bewcgung der Elektronen wird hochgradig irregulär oder diffusiv, genauso wie bei der Brownschen Bewegung. Theoretische Rechnungen sagen daher eine $\mathrm{Ab}$ schwächung des Daucrstroms, im Vergleich zum idealen Ring, um einen Faktor voraus, der gerade durch das Verhältnis aus mittlerer freier Weglänge zu Umfang gegeben ist. Wic bereits diskutiert, ergibt sich sowohl eine Halbierung der Periode in der Abhangigkeit rom magnetischen Fluß, als auch eine weitere Absenkung der Größe des Effekts, bei Mittetheoretischen Ergebnisse sind im Vergleich zu den vorliegenden experimentellen Beobachlung über eine Gesamtheit von Ringen. Beide 
tungen um etwa zwei Größenordnungen zu klein!

In verschiedenen Erwciterungen der Theorie wurde schließlich noch der Einfluß der Wechselwirkung zwischen den Elektronen untersucht. Damit wird ein hochaktuelles, aber auch sehr schwieriges Gebiet betreten.
Wie verhalten sich quantenmechanische Interferenzen, wenn gleichzeitig zufällige $\mathrm{De}$ fekte und die Wechselwirkung zwischen den geladenen Teilchen eine Rolle spielen? Diese Frage ist heute noch weitgehend offen. Bei den Dauerströmen zeigte sich, daß die Wechselwirkung einen wichtigen Beitrag für eine Gesamtheit vieler Ringe liefert, der aber für eine Erklärung der Diskrepanz zwischen den Experimenten und der Theorie ebenfalls zu klein ist. Wechselwirkungsbeiträge zum Strom in einzelnen Ringen sind noch weitgehend unverstanden.

Mit großer experimenteller Anstrengung ist es gelungen, winzige Dauerströme in meso-

\section{Quantenmechanik eines eindimensionalen Ringes}

Wir betrachten einen exakt eindimensionalen Draht der Länge $L$ entlang der $x$-Achse, $0 \leq \mathrm{x} \leq L$. Die möglichen Energieniveaus eines Teilchens sind durch Lösung der stationären Schrödinger-Gleichung unter bestimmten Randbedingungen zu bestimmen. Diese lautet $(\hbar=h / 2 \pi)$

$$
-\frac{\hbar^{2}}{2 m} \psi^{\prime \prime}(x)+V(x) \psi(x)=\varepsilon \psi(x)
$$

Hier ist $\psi(x)$ die komplexe Wellenfunktion, $\psi^{\prime}=d \psi / d x$, und $V(x)$ die potentielle Energie. Wir setzen zur Vereinfachung $V(x) \equiv 0$.

\section{Der Ansatz}

$\psi(x)=A e^{i k x}+B e^{-i k x}$

mit zwei Konstanten $A$ und $B$ führt zu

$\varepsilon=\hbar^{2} k^{2} / 2 m$

Diese Beziehung ist der klassische EnergieImpuls-Zusammenhang, wenn wir den Impuls $p=\hbar k$ einführen. Wir stellen uns nun vor, daß der Draht zu einem Ring verformt wird. Dann muß die Wellenfunktion bei $x=$ 0 und $x=L$ gleich sein (periodische Randbedingungen). Aus $\psi(0)=\psi(L)$ folgt, daß der Wellenvektor $k$ nur bestimmte Werte annehmen kann, nämlich

$k=\frac{2 \pi}{L} n$

mit $n=0, \pm 1, \pm 2, \ldots$ Eine Verallgemeinerung dieser Randbedingung ist von der Form $\psi(0)=\psi(L) e^{\mathrm{i} \varphi}$, woraus

$k=\frac{2 \pi}{L}\left(n-\frac{\varphi}{2 \pi}\right)$ folgt. Die Energieniveaus sind durch Gleichung (3) bestimmt.

Die obigen Ergebrisse geben auch an, wie die Energieniveaus eines Rings im Magnetfeld vom magnetischen Fluß $\Phi$ abhängen, wenn wir $\varphi$ mit $2 \pi \Phi / \Phi_{0}$ identifizieren. Wegen $\mathbf{B}=\nabla \times \mathbf{A}$ läßt sich für ein konstantes Magnetfeld das Vektorpotential A als $\mathbf{B} \times \mathbf{r} / 2$ darstellen. In der betrachteten Geometrie zeigt B senkrecht zur Ringebene, $\mathbf{r}$ liegt in der Ringebene, und $\mathbf{A}$ ist somit entlang des Rings gerichtet und betragsmäßig durch $B r / 2=B L / 4 \pi$ gegeben. Dann verwenden wir die klassische Beziehung $\varepsilon=m v^{2} / 2$, wobei die Geschwindigkeit $v$ mit dem Wellenvektor gemäß $v=\hbar k-e A / m$ ) verknüpft ist, sowie die Quantisierungsbedingung (4) und $\Phi=B L^{2} / 4 \pi$. Dies führt ebenfalls zu dem angegebenen Ergebnis.

Offensichtlich ist die Gesamtheit des Spektrums periodisch in $\Phi / \Phi_{0}$ mit Periode 1, so $\mathrm{da} ß$ wir uns auf den Bereich $-1 / 2 \ldots+1 / 2$ beschränken können. Das Ergebnis ist in der Abbildung als gestrichelte Linie gezeichnet. Durch Ableitung nach dem magnetischen Fluß finden wir den Dauerstrom, der von einem Energieniveau getragen wird. Schließlich verwenden wir noch das Pauli-Prinzip (Elektronen sind Fermionen!), nach dem jeder Zustand nur von einem Elektron besetzt werden darf, und berechnen den gesamten Dauerstrom als Summe der von den einzelnen Niveaus getragenen Ströme. Betrachten wir als Beispiel drei im Vergleich zu vier Elektronen für $\Phi \approx 0$. Offensichtlich tragen drei Elektronen keinen Strom, während sich das vierte Elektron entscheiden muß, ob es einen Zustand mit positivem oder negativem Impuls besetzen soll. Diese Entartung wird für $\Phi \neq 0$ aufgehoben, was zu einem Sprung in
$I(\Phi)$ für eine gerade Zahl von Elektronen führt.

Schon eine geringe $Z$ ahl von Defekten führt zur Bildung von Energielücken (siehe $\mathrm{Ab}$ bildung, durchgezogene Linien) mit dem Ergebnis, daß Unstetigkeiten in $I(\Phi)$ verschmiert werden. Das gezeigte Spektrum hat - nicht nur zufällig - Ähnlichkeiten mit der Bandstruktur eines Festkörpers.

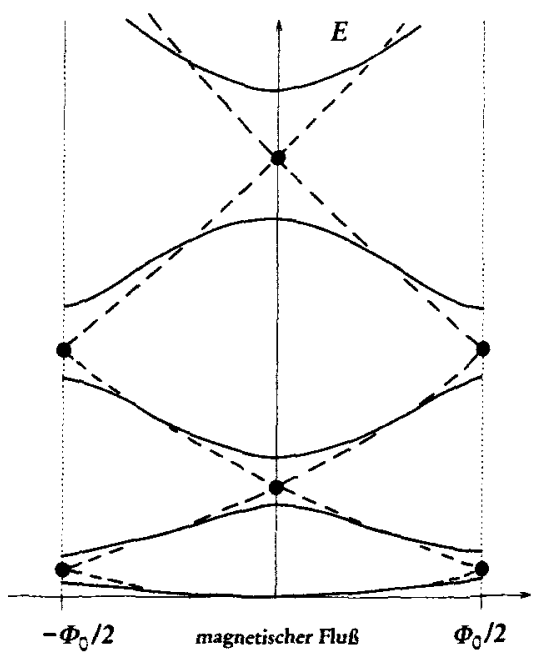

Schematische Darstellung der Energieniveaus als Funktion des magnetischen Flusses unter Berücksichtigung von Unordnung (durchgezogene Linien). Im idealen Fall (keine Defekte, gestrichelte Linien) verschwinden die Energielücken, und die Ableitung nach $\Phi$ ist unstetig bei $\Phi=0$ und $\Phi= \pm \Phi_{0} / 2$. 


\section{Normale Dauerströme}

Allgemeine Überlegungen zeigen, daß für ringartige Strukturen verschiedene physikalische Größen Interferenzeffekte zeigen und daher vom eingeschlossenen magnetischen Fluß abhängen. Als Beispiel betrachten wir die Gesamtenergie der Elektronen,

$E(\Phi)=E_{0}+E_{1}(\Phi)$

Ein typischer mesoskopischer Ring enthält etwa $10^{10}$ Elektronen, somit ist $E_{0}$ eine Konstante von der Größenordnung $10^{10} \mathrm{eV}$, während $E_{1}(\Phi)$ eine im Vergleich dazu winzige, flußabhängige Korrektur darstellt. Mit dieser Flußabhängigkeit ist aber ein Strom verknüpft,

$I(\Phi)=-\frac{d E(\Phi)}{d \Phi}$

den wir als Dauerstrom bezeichnen wollen. Diesem Dauerstrom entspricht eine Magnetisierung $M=-d E / d B$, wobei $B=4 \pi \Phi / L^{2}$ der Betrag des Magnetfeldes und $L$ der Umfang ist ( $M$ und $B$ sind senkrecht zur Ringebene gerichtet).

Ausgehend von einem allgemeinen Prinzip, der Zeitumkehrinvarianz, läßt sich nun eine wichtige Schlußfolgerung ziehen. Danach ist die Energie eine gerade Funktion des Magnetfeldes, wenn das Metall keine magnetischen Fremdatome enthält. Das ist auch anschaulich recht sinnvoll. Folgerichtig ist $I(\Phi)$ eine ungerade Funktion von $\Phi$. Somit können wir ansetzen

$$
I(\Phi)=I_{1} \sin \left(2 \pi \frac{\Phi}{\Phi_{0}}\right)+I_{2} \sin \left(4 \pi \frac{\Phi}{\Phi_{0}}\right)+\ldots
$$

welches eine Entwicklung nach Harmonischen darstellt. Die experimentellen Beobachtungen [2-4] deuten darauf hin, daß der Koeffizient $I_{1}$ in dieser Entwicklung außerordentlich sensitiv von der mikroskopischen Konfiguration der Defekte abhängt und insbesondere von Ring zu Ring das Vorzeichen wechseln kann. Daher ergibt sich im Mittel über viele Ringe [2] der Wert Null, die führende Periode in der Abhängigkeit vom magnetischen Fluß ist nicht $\Phi_{0}$, sondern durch den zweiten Beitrag gegeben und damit $\Phi_{0} / 2$. An einzelnen Ringen $[3,4]$ ist die Periode $\Phi_{0}$ deutlich sichtbar. skopischen Normalmetallringen zu beobachten. Nach allem, was wir heute wissen, lassen sich diese Effekte nur mit Hilfe von quantenmechanischen Interferenzen, die sich bei tiefen Temperaturen über Abstände von bis $\mathrm{zu}$ hunderttausend Atomdurchmessern erstrecken können, crklären. Aber, trotz etwa siebzig Jahren erfolgreicher Anwendung der Quantenmechanik in zahlreichen Bereichen der Physik, wie beispielsweise der Atom-, Molekül- und Festkörperphysik, steht zur Zeit eine befriedigende quantitative $\mathrm{Er}$ klärung der experimentell beobachteten Effekte noch aus - der gemessene Dauerstrom ist um zwei Größenordnungen größer als theoretisch vorhergesagt! Das zeigt uns sehr deutlich, daß unser Verständnis der quantenmechanischen Theorie kohärenter Systeme mit vielen Freiheitsgraden noch immer sehr lückenhaft und noch lange nicht ausgereift ist, und das ist tatsächlich eine Überraschung, nachdem es in den vergangenen zehn Jahren gelungen war, eine große Zahl von Quanteninterferenzeffekten in ähnlichen Systemen sogar quantitativ zu verstehen.

\section{Literatur}

[1] A. Gladun, A. B. Zorin, Physik in unserer Zeit 23, 159 (4/1992).

[2] L. P. Levy et al., Phys. Rev. Lett. 64, 2074 (1990); L. P. Levy, Physica B 169, 245 (1991).

[3] V. Chandrasekhar et al., Phys. Rev. Lett. 67, 3578 (1991).

[4] D. Mailly, C. Chapelier, A. Benoit, Phys. Rev. Lett. 70, 2020 (1993).

[5] M. Büttiker, Y. Imry, R. Landauer, Phys. Lett. 96A, 365 (1985).

[6] U. Eckern, PTB-Mitteilungen 101, 259 $(4 / 1991)$

[7] L. P. Levy, Physics Today 45, 17 (4/1992).

[8] U. Eckern und A. Schmid, Ann. Physik (Leipzig) 2, 180 (1993).

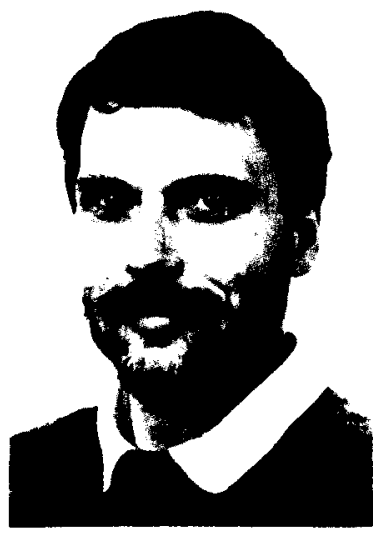

Ulrich Eckern, geb. 9. 3. 1952, Studium der Physik in Dortmund und Karlsruhe, danach Post-doc an der Cornell University, Assistent und Heisenberg-Stipendiat an der Universität Karlsruhe und wissenschaftlicher Mitarbeiter am Kernforschungszentrum Karlsruhe, seit April 1993 Ordinarius für Theoretische Physik an der Universität Augsburg.

\section{Anschrift:}

Prof. Dr. U. Eckern, Lehrstuhl für Theoretische Phvsik II, Universitat Augsburg. $11 \mathrm{~cm}$ minger Str. 6, 86135 Augsburg. 